\title{
Analysis of Propagation of Electromagnetic Waves in Atmospheric Hydrometeors on Low-Elevation Paths
}

\author{
Martin GRABNER ${ }^{1}$, Pavel PECHAC ${ }^{2}$, Pavel VALTR ${ }^{2}$ \\ ${ }^{1}$ Dept. of Frequency Engineering, Czech Metrology Institute, Okruzni 31, 63800 Brno, Czech Republic \\ 2 Dept. of Electromagnetic Field, Faculty of Electrical Engineering, Czech Technical University in Prague, \\ Technicka 2, 16627 Praha 6, Czech Republic
}

mgrabner@cmi.cz, \{pechac, pavel.valtr\}@fel.cvut.cz

Submitted January 29, 2017 / Accepted October 18, 2017

\begin{abstract}
Attenuation of electromagnetic waves in millimeter wave bands is analyzed by means of experimental measurement of received signal fluctuations on terrestrial radio links operating in frequency bands 58, 94 and $122 \mathrm{GHz}$. Long-term time series of the received signal are processed to obtain annual and two-year cumulative distributions of attenuation due to hydrometeors. The measured statistics give the attenuation higher than predicted by the model of the Recommendation ITU-R P.530. Rain intensity measured simultaneously with rain attenuation is used to obtain fitted parameters of an attenuation/intensity power-law relationship. The empirical data extracted from the experiment are compared with the results of numerical simulations of attenuation due to rain and hailstones.
\end{abstract}

\section{Keywords}

Electromagnetic wave propagation, rain attenuation, scattering

\section{Introduction}

Wireless communications are steadily shifting toward utilization of higher frequency bands that can satisfy an increasing transmission capacity demands. Terrestrial and low elevation links in particular operating in millimeter wave bands are expected to be utilized more frequently in foreseeable future. Electromagnetic waves of millimeter wavelengths propagating in atmosphere are mostly impaired by atmospheric hydrometeors [1] due to scattering/absorption of $\mathrm{mm}$ waves on droplets forming a hydrometeor. In order to estimate the quality and availability of the designed mm-wave radio links, the statistics of attenuation are thus needed [2]. Nevertheless long-term empirical statistics of attenuation are not widely available in literature so far, especially for frequencies around and above $100 \mathrm{GHz}$.

Attenuation due to atmospheric hydrometeors is known to be proportional to the intensity of precipitation. However the incidence of precipitations is locally specific and has to be determined experimentally [3-5]. The goal of this work is therefore to obtain so far not unavailable empirical local statistic of attenuation that can be used 1) to assess propagation models and 2) to design mm wave links in the area. Furthermore, the dependence of $\mathrm{mm}$ wave attenuation on the physical parameters of hydrometeors such as rain intensity is studied. The experimental measurement of the received signal fluctuations have been carried out simultaneously on three mm-wave links [6], [7]. The statistics of attenuation have been obtained from the long-term dataset.

\section{Experimental Setup}

Experimental observation of path attenuation fluctuations is carried out continuously on several millimeter wave links with receiver site located at the Czech Metrology Institute (CMI) building (Hvozdanska street) in Prague, see Fig. 1.

The two links operating at $58 \mathrm{GHz}$ and $122 \mathrm{GHz}$ [8] on the same path with the transmitters located on the rooftop of the Senohrabska street (Sen) building are almost perpendicular to the $94 \mathrm{GHz}$ link with the transmitter located on the building of the Geophysical Institute (GFU) (Bocni II street). Table 1 summarizes basic parameters of mm links.

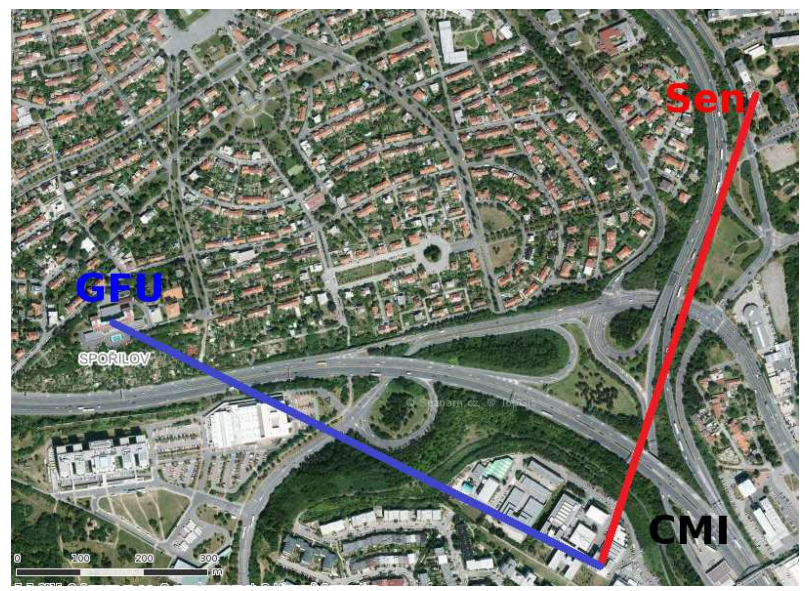

Fig. 1. The map of experimental microwave wave paths orientation. 


\begin{tabular}{|l|r|r|r|}
\hline Link no. & $\mathbf{1}$ & $\mathbf{2}$ & $\mathbf{3}$ \\
\hline Frequency [GHz] & 58.15 & 94.63 & 122.25 \\
\hline Path length [m] & 750 & 853 & 750 \\
\hline Elevation angle [deg] & 1.9 & 2.2 & 1.9 \\
\hline Antenna size/diameter $[\mathrm{cm}]$ & $20 \times 30$ & 30 & 24 \\
\hline Transmitted power $[\mathrm{dBm}]$ & 5 & 17 & -1.55 \\
\hline Approx. fade margin $[\mathrm{dB}]$ & 16 & $>26$ & $>40$ \\
\hline
\end{tabular}

Tab. 1. Parameters of experimental millimeter wave links.

An automatic gain control (AGC) voltage is measured using an $\mathrm{A} / \mathrm{D}$ converter on a $\mathrm{PC}$ card and received signal level (RSL) is calculated via calibration curves that were obtained during the calibration before a radio link deployment. The 58 and $94 \mathrm{GHz}$ links were calibrated at the CMI by means of precise attenuators connected between a transmitter and receiver via a rectangular waveguide. The $122 \mathrm{GHz}$ link was calibrated by its manufacturer [8].

The nominal received signal level was determined dominantly as a monthly median value of the RSL such that the nominal RSL is taken as a zero attenuation level. In some particular cases of fading events on the $122 \mathrm{GHz}$ link, the zero level was obtained from the RSL immediately before and after the individual attenuation event.

Beside other relevant meteorological quantities, rain intensity is measured simultaneously near the receivers by means of two tipping bucket heated rain gauges with sensitivities: $0.1 \mathrm{~mm} /$ hour per tip and $0.2 \mathrm{~mm} /$ hour per tip respectively [6]. Two additional microwave links in the frequency band $58 \mathrm{GHz}$ with $\mathrm{V}$ and $\mathrm{H}$ polarizations are observed on the path GFU-CMI. The links provided rain attenuation data as presented below in Fig. 2.

\section{Experimental Results}

\subsection{Attenuation Statistics}

Attenuation statistics from the period from May 2013 to April 2014 were presented in [7]. More than 100 hydrometeor events were individually selected and cumulative distributions (i.e. percentages of time when attenuation exceeded a given level) of (specific) attenuation given in $(\mathrm{dB} / \mathrm{km})$ were obtained. Annual cumulative distributions obtained from the period from May 2014 to April 2015 are shown in Fig. 2.

The cumulative distributions of attenuation obtained from the whole two-year period from May 2013 to April 2015 are shown in Fig. 3.

The annual distributions are compared with the predicted ones using the Recommendation ITU-R P.530 [9]. P.530 applies the value of rain intensity $R_{01}$ exceeded for $0.01 \%$ of time at a particular location to predict rain attenuation statistics at that location. Two sets of distributions depicted are predicted using particular values $R_{01}=24 \mathrm{~mm} / \mathrm{h}$ and $R_{01}=32 \mathrm{~mm} / \mathrm{h}$. The former value is obtained from ITU$\mathrm{R}$ world maps [10], the latter is obtained from long-term local measurements of rain in the climate of $\mathrm{CR}$. The reasonable

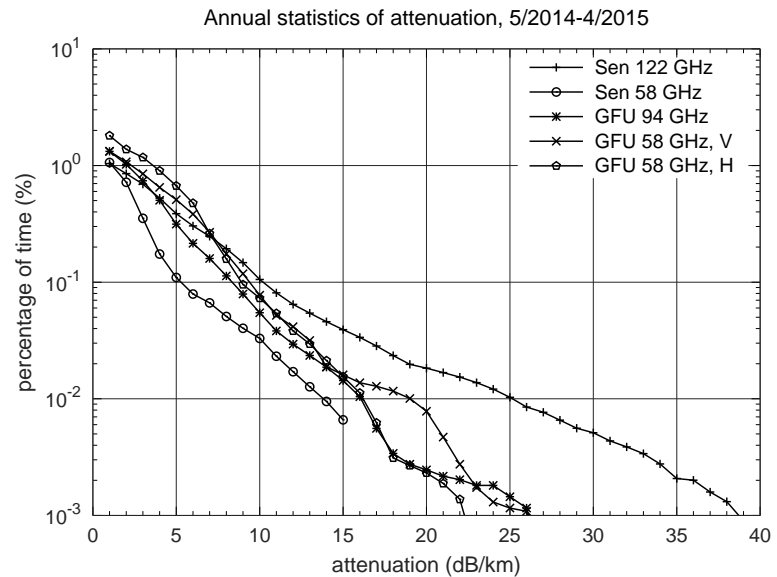

Fig. 2. Annual cumulative distributions of attenuation due to hydrometeors

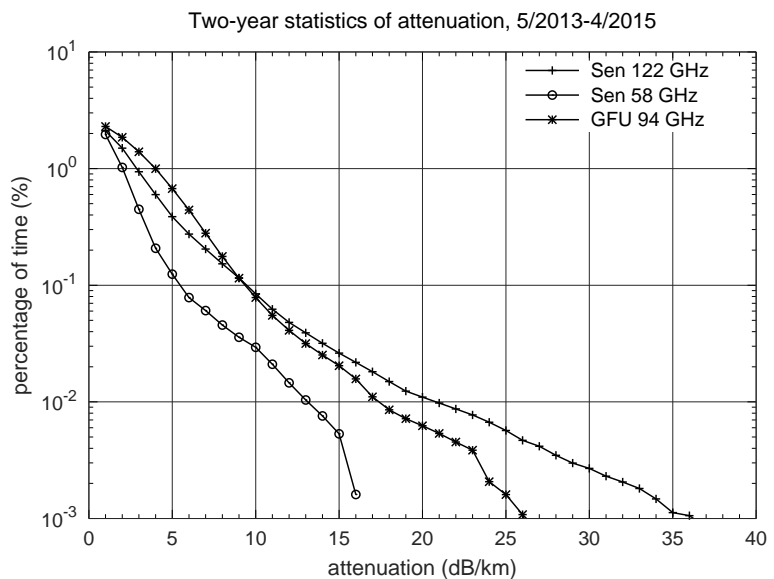

Fig. 3. Two-year cumulative distributions of attenuation due to hydrometeors.

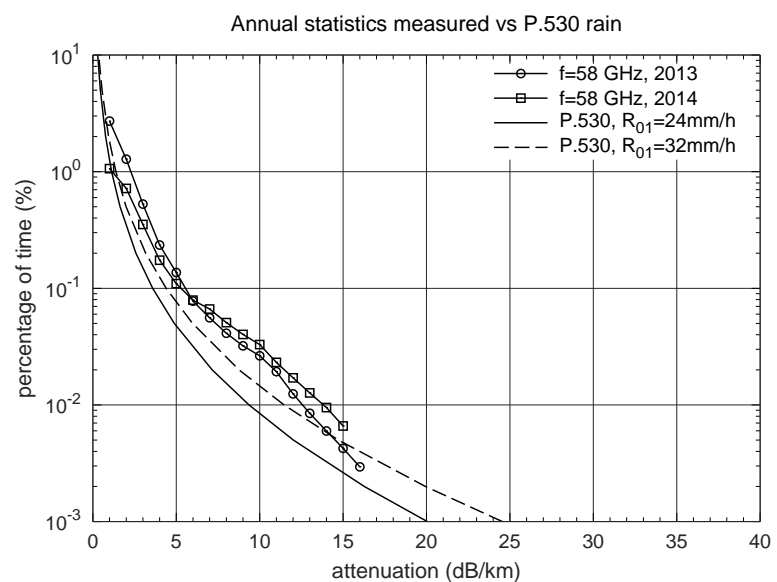

Fig. 4. Predicted (ITU-R P.530) and measured annual cumulative distributions of attenuation due to hydrometeors.

agreement between measured and predicted distributions is seen but the measured attenuation seems to be systematically higher than the predicted one. One must also take the year-to-year variability into account. 


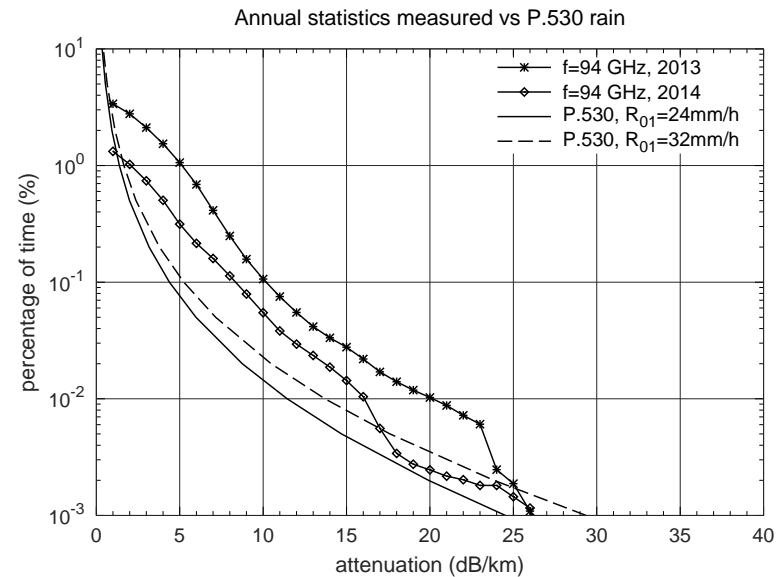

Fig. 5. Predicted (ITU-R P.530) and measured annual cumulative distributions of attenuation due to hydrometeors

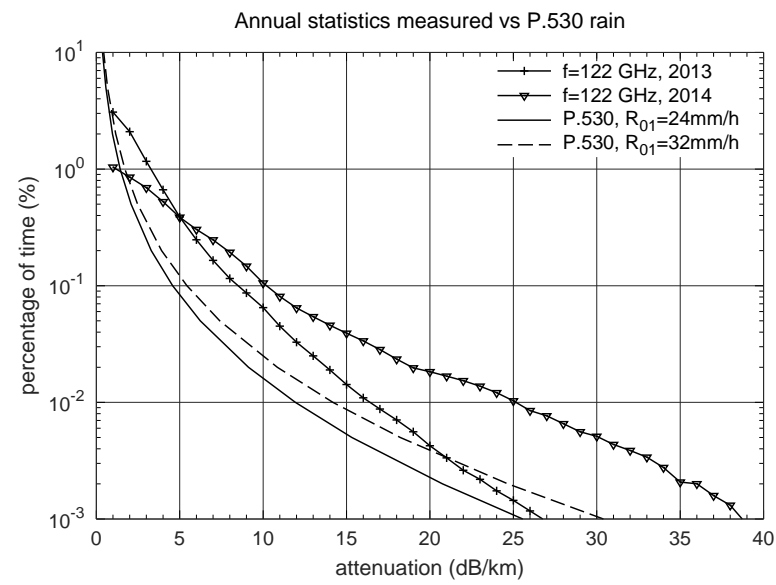

Fig. 6. Predicted (ITU-R P.530) and measured annual cumulative distributions of attenuation due to hydrometeors.

\subsection{Attenuation vs Rain Intensity}

Figure 7 shows a scatter plot of two-year data of simultaneously observed attenuation and rain intensity. The data depicted are the average values over the 20 second time intervals. This averaging length serves as a compromise between typical attenuation sampling ( $1 \mathrm{sec}$.) and a usual rain intensity averaging interval (1 min.).

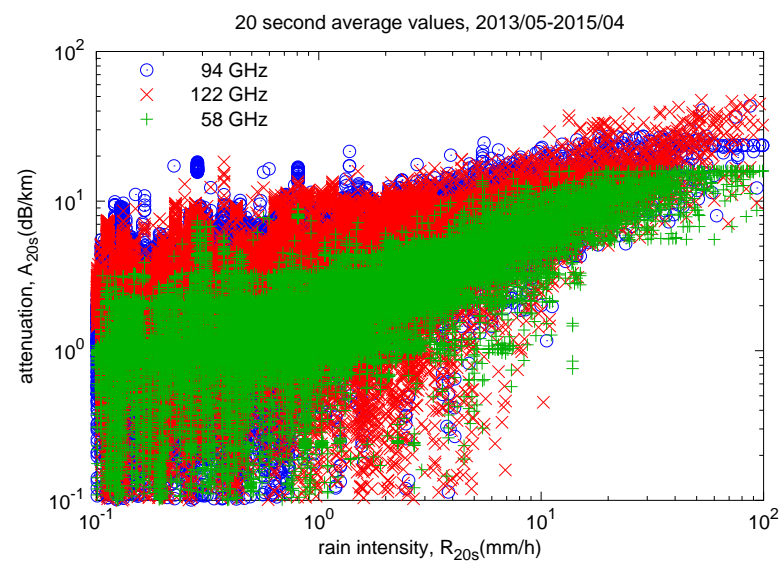

Fig. 7. Scatter plot of rain attenuation vs intensity.

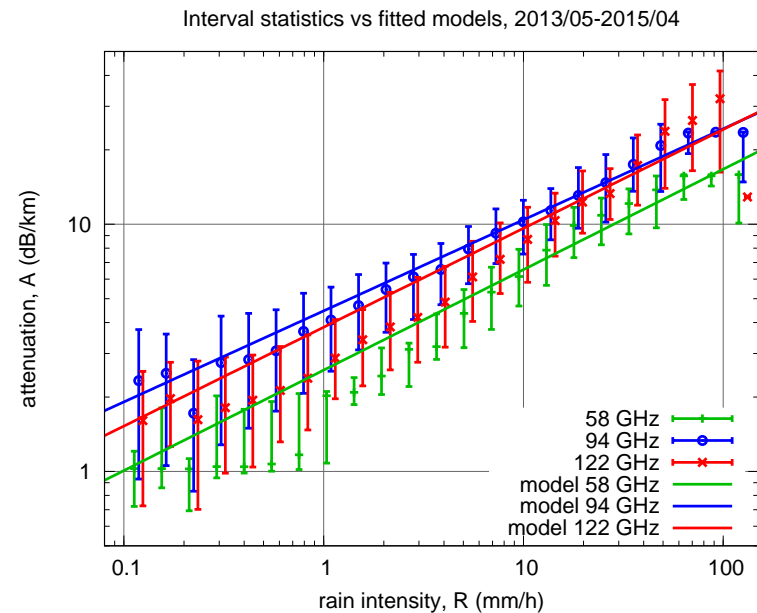

Fig. 8. Interval statistics (percentiles: lower boundary 20\%, median point $50 \%$, upper boundary $80 \%$ ) of attenuation vs intensity and fitted power law models.

Figure 8 show interval statistics obtained from the scatter plot (see above) by dividing a full rain intensity range into subintervals and calculating statistics of attenuation data falling into each subinterval.

Furthermore, the power law models of the form

$$
A=4.34 \times k R^{q}
$$

where $A(\mathrm{~dB} / \mathrm{km})$ is specific attenuation and $k, q$ are the parameters of the model, are fitted to the interval median values (i.e. $50 \%$ percentiles) of attenuation. Table 2 summarizes fitted parameters of the model. Note that only $R>1 \mathrm{~mm} / \mathrm{h}$ values were actually fitted since the region of low attenuation/intensity is relatively less important in practical applications. There is also a larger relative measurement error in this region.

\begin{tabular}{|l|r|r|r|}
\hline Link no. & $\mathbf{1}$ & $\mathbf{2}$ & $\mathbf{3}$ \\
\hline Frequency $[\mathrm{GHz}]$ & 58.15 & 94.63 & 122.25 \\
\hline$k$ & 0.591 & 1.028 & 0.883 \\
\hline$q$ & 0.406 & 0.368 & 0.400 \\
\hline
\end{tabular}

Tab. 2. Parameters of fitted power law models (1).

\section{Scattering Modeling}

The measurement results, specific for 3 millimeter wave bands, presented in the previous section is to be confronted with the numerical modeling predictions. Specifically, the frequency dependence attenuation due to scattering on the water particles (rain) and on ice particles (hailstones) is investigated in the following. The specific attenuation $\mathrm{A}(\mathrm{dB} / \mathrm{km})$ is obtained by integrating droplet extinction cross sections $C_{\text {ext }}$ weighted by droplet size distribution (DSD) (3) [11], [12] as follows:

$$
A=4.34 \int_{0}^{\infty} C_{\text {ext }}(r, \lambda, m) n(r) \mathrm{d} r
$$


where the cross section is a function of the droplet radius $r$, the wavelength $\lambda$ and of the complex refractive index of water or ice $m$. Consistent units are assumed in (2). The extinction cross section $C_{\text {ext }}$ is calculated using Mie scattering formulas [11], [13]. A gamma distribution is used which is common to describe the realistic DSD of real hydrometeors

$$
n(r)=a r^{\alpha} \exp (-b r)
$$

where the parameters $a, \alpha$ and $b$ depend on the physical parameters of a particular hydrometeor. Several models have been proposed for DSD of rain parametrized by rain intensity. The widely used classical Marshal-Palmer (MP) model [14] is defined by an exponential distribution which is a special case of (3) with $\alpha=0$. A slightly modified MP model is used here with the parameters of the gamma model (3) are $a=16000 \mathrm{~mm}^{-1} \mathrm{~m}^{-3}, \alpha=0.5$ and $b=8 R^{0.2} \mathrm{~mm}^{-1}$. In this case, $n(r)$ has units $\mathrm{mm}^{-1} \mathrm{~m}^{-3}$ and the drop radius is expressed in millimeters.

The attenuation calculated using numerical integration of (2) is shown in Fig. 9. The results of theoretical calculation seems to be fully consistent with the results of measurement in three $\mathrm{mm}$ wave bands presented in previous sections. Modeling of scattering on ice particles show that with the same DSD attenuation attenuation due to hailstones is lower than the rain attenuation but only for frequencies lower than $100-150 \mathrm{GHz}$

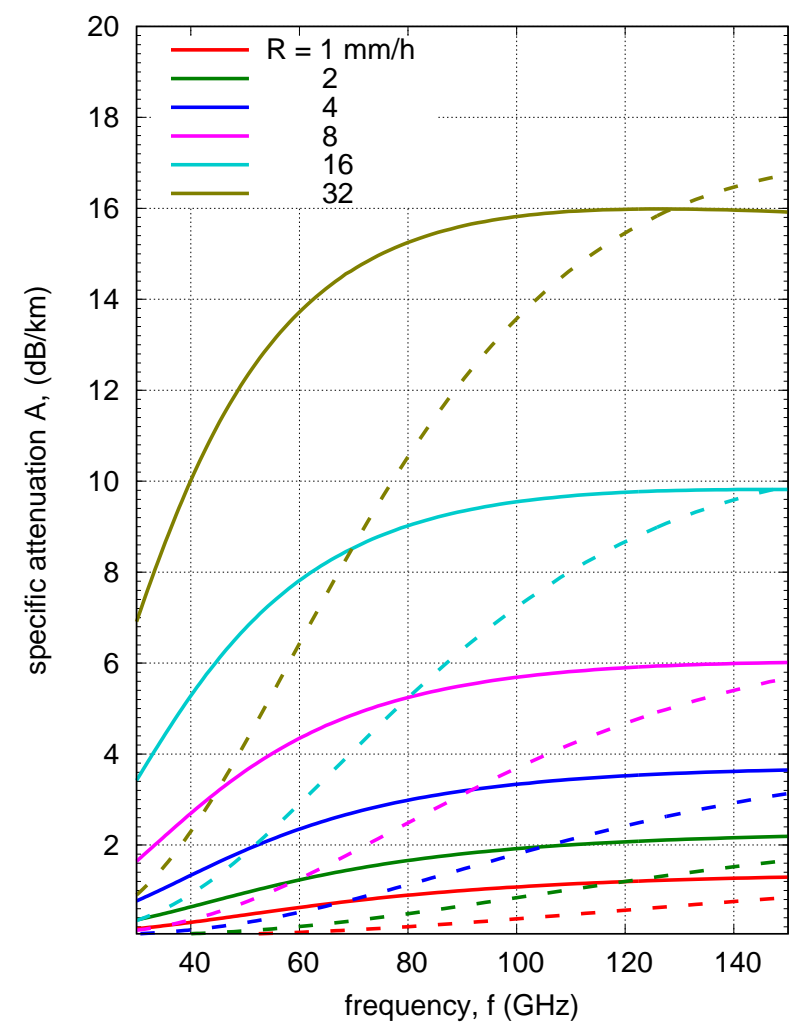

Fig. 9. Frequency dependence of rain (solid) and hailstone (dashed) attenuation obtained by Mie scattering calculations for different rain intensities.

\section{Conclusion}

The long-term statistics of attenuation due to hydrometeors in three $\mathrm{mm}$ wave bands were obtained from the measured received power levels on terrestrial microwave links in Prague. The measured attenuation levels are slightly larger than predicted by the ITU-R P.530. This may be caused by a higher incidence of stronger rain events.

Attenuation observed in all three frequency bands under study was generally of very similar level and with estimated measurement accuracy of the order $1-3 \mathrm{~dB}$ it is difficult to distinguish different frequencies even on the same path. Nevertheless, attenuation of $58 \mathrm{GHz}$ band is consistently lower than attenuation of two higher frequency bands, this fact is confirmed also by a numerical model in Fig. 9. On the other hand the difference between 94 and $122 \mathrm{GHz}$ seems to be less significant. The observed attenuation on the $94 \mathrm{GHz}$ link is actually slightly higher than on the $122 \mathrm{GHz}$ link which seems to be in contrast with the theoretical predictions in Fig. 9. However one must note that a) their propagation paths are different (perpendicular) and b) the measurement accuracy of the order 1-3 dB mentioned above has to be taken into account. Therefore one can conclude that the trend of increasing attenuation due to hydrometeors with increasing frequency is limited to frequencies below about $100 \mathrm{GHz}$ and attenuation does not increase significantly in higher $\mathrm{mm}$ wave bands.

Rain and snow attenuation was not specifically distinguished in our experiment in a sense but the attenuation events estimated to be due to snow covering antennas were removed from the processing. However pure snow events usually caused attenuation of several $\mathrm{dB}$ and the strongest attenuation events were caused by a heavy rain as was checked by comparison with concurrent on-site meteorological measurements.

\section{Acknowledgments}

This work was financially supported by the Czech Science Foundation grant no. P102/14-01527S

\section{References}

[1] KERR, E. Propagation of Short Radio Waves. 2nd ed. London (UK): Peter Peregrinus, 1987. ISBN: 0863410995

[2] KIZER, G. Digital Microwave Communication. 1st ed. New Jersey (USA): IEEE Press \& Wiley, 2013. ISBN: 9780470125342

[3] MANABE, T., IHARA, T., AWAKA, J., et al. The relationship of raindrop-size distribution to attenuations experience at 50, 80, 140, and $240 \mathrm{GHz}$. IEEE Transactions on Antennas and Propagation, 1987, vol. 35, no. 11, p. 1326-1330. DOI: 10.1109/TAP.1987.1144005

[4] HIRATA, A., YAMAGUCHI, R., TAKAHASHI, H., et al. Effect of rain attenuation for a 10-Gb/s 120-GHz-band millimeter- 
wave wireless link. IEEE Transactions on Microwave Theory and Techniques, 2009, vol. 57, no. 12, p. 3099-3105. DOI: 10.1109/TMTT.2009.2034342

[5] HONG, E., LANE, S., MURRELL, D., et al. Terrestrial link rain attenuation measurements at $84 \mathrm{GHz}$. In Proceedings of the United States National Committee of URSI National Radio Science Meeting (USNC-URSI NRSM). Boulder (USA), 2017, p. 1-2. DOI: 10.1109/USNC-URSI-NRSM.2017.7878267

[6] GRABNER, M., PECHAC, P., VALTR, P. First results of concurrent rain attenuation measurement in three millimeter wave bands. In Proceedings of the XXXIth URSI General Assembly and Scientific Symposium. Beijing (China), 2014, p. 1-4. DOI: $10.1109 /$ URSIGASS.2014.6929689

[7] GRABNER, M., PECHAC, P., VALTR, P. Attenuation due to hydrometeors in three millimeter wave bands. In Proceedings of the 10th European Conference on Antennas and Propagation (EuCAP). Davos (Switzerland), 2016, p. 1-4. DOI: 10.1109/EuCAP.2016.7481331

[8] KOLKA, Z., KINCL, Z., BIOLKOVA, V., et al. Hybrid FSO/RF test link. In Proceedings of the 4th International Congress on $\mathrm{Ul}$ tra Modern Telecommunications and Control Systems and Workshops (ICUMT). St. Petersburg (Russia), 2012, p. 502-505. DOI: 10.1109/ICUMT.2012.6459718

[9] ITU RADIOCOMMUNICATION SECTOR Rec. ITU-R P.530-16, Propagation data and prediction methods required for the design of terrestrial line-of-sight systems. Geneva (Switzerland): ITU, 2015.

[10] ITU RADIOCOMMUNICATION SECTOR Rec. ITU-R P.837-6, Characteristics of precipitation for propagation modelling. Geneva (Switzerland): ITU, 2012.
[11] VAN DE HULST, H. C. Light Scattering by Small Particles. 1st ed. New York (USA): Dover Publications, 1981. ISBN: 0486642283

[12] ISHIMARU, A. Wave Propagation and Scattering in Random Media. 2nd ed. Piscataway (USA): IEEE Press, 1997. ISBN: 078034717X

[13] WISCOMBE, W. J. Improved Mie scattering algorithms. Applied $O p$ tics, 1980, vol. 19, no. 9, p. 1505-1509. DOI: 10.1364/AO.19.001505

[14] MARSHALL, J. S., PALMER, W. M. The distribution of raindrops with size. Journal of Meteorology, 1948, vol. 5, no. 4, p. 165-166. DOI: 10.1175/1520-0469(1948)005<0165:TDORWS>2.0.CO;2

\section{About the Authors...}

Martin GRÁBNER received his M.Sc. and Ph.D. from the Czech Technical University in Prague in 2000 and 2008. His research interests include electromagnetic wave propagation in atmosphere.

Pavel PECHAČ received his M.Sc. and Ph.D. from the Czech Technical University in Prague. His research interests include electromagnetic wave propagation.

Pavel VALTR received his M.Sc. and Ph.D. from the Czech Technical University in Prague. His research interests include electromagnetic wave propagation. 